\title{
Attractive Hofstadter-Hubbard model with imbalanced chemical and vector potentials
}

\author{
M. Iskin \\ Department of Physics, Koç University, Rumelifeneri Yolu, 34450 Sartyer, Istanbul, Turkey
}

(Received 9 January 2015; published 7 May 2015)

\begin{abstract}
We study the interplay between the Hofstadter butterfly, strong interactions, and the Zeeman field within the mean-field Bogoliubov-de Gennes theory in real space, and explore the ground states of the attractive single-band Hofstadter-Hubbard Hamiltonian on a square lattice, including the exotic possibility of imbalanced vector potentials. We find that the cooperation between the vector potential and superfluid order breaks the spatial symmetry of the system, and stripe-ordered Fulde-Ferrell-Larkin-Ovchinnikov-like superfluid and supersolid phases occur that can be distinguished and characterized according to their coexisting pair-density-, chargedensity-, and spin-density-wave orders. We also discuss confined systems and comment on the likelihood of observing such stripe-ordered phases by loading neutral atomic Fermi gases on laser-induced optical lattices under laser-generated artificial gauge fields.
\end{abstract}

DOI: 10.1103/PhysRevA.91.053606

PACS number(s): 03.75.Ss, 03.75.Hh, 67.85.- d, 67.80.kb

\section{INTRODUCTION}

The exact energy spectrum of a single quantum particle that is confined to move on a two-dimensional tight-binding periodic lattice under the influence of a uniform magnetic flux has been known for a long time [1,2], where the competition between the lattice spacing and cyclotron radius gives rise to a self-similar complex pattern of sub-bands and minigaps. However, in spite of all efforts since the prediction of this Hofstadter spectrum, there has only been very recent but still limited success in observing some of its signatures in graphene-based solid-state materials with artificially engineered superlattices under real magnetic fields $[3,4]$. In addition, thanks to the recent realization of artificial gauge fields in atomic systems [5-12], there is also an increasing interest in this subject from the cold-atom community [13-20]. In particular, by engineering spatially dependent complex tunneling amplitudes with laser-assisted tunneling and a potential energy gradient, two research groups have recently reported realization of the Hofstadter-Harper Hamiltonian using neutral rubidium atoms that are loaded into laser-induced periodic potentials [16-18].

Even though the Hofstadter and Hubbard Hamiltonians have themselves been the subject of many works in the literature, there has been a lack of interest in the combined Hofstadter-Hubbard Hamiltonian even at the mean-field level. For instance, while the use of momentum-space BCS formalism limits previous analysis of the attractive HofstadterHubbard model to vortex lattice (VL) configurations only [21], the existence of pair-density-wave (PDW) and VL orders has been proposed in the context of a somewhat related model: an anisotropic three-dimensional (3D) continuum Fermi gas experiencing a uniform magnetic flux [22]. By first limiting their description to the lowest-Landau-level limit and then making further assumptions about the strength of the anisotropic trap, the authors obtain an effectively 1D Hamiltonian in momentum space, and solved it using the BCS formalism. The existence and characterization of a variety of distinct stripe-ordered many-body phases appear to have either been overlooked or gone unnoticed until very recently [23], distinguishing our work from the literature.

In particular, here we use Bogoliubov-de Gennes (BdG) theory in real space and study the mean-field ground states of the attractive single-band Hofstadter-Hubbard Hamiltonian on a square lattice, including the effects of imbalanced chemical and vector potentials. We find that the cooperation between the vector potentials and interaction breaks the spatial symmetry of the system, leading to various stripe-ordered superfluid (SF) and supersolid (SS) phases that can be distinguished and characterized according to their coexisting PDW, chargedensity- (CDW), and spin-density- (SDW) wave orders. We also discuss possible observation of such stripe-ordered phases by confining neutral atomic Fermi gases in laser-induced optical lattices under laser-generated artificial gauge fields.

The rest of this paper is organized as follows. In Sec. II, first we introduce the physical setting of the problem and the model Hamiltonian used, then review the noninteracting Hofstadter Hamiltonian and its well-known Hofstadter spectrum, and then describe the self-consistent BdG formalism which takes fermion-fermion interactions into account within the meanfield approximation for pairing. The resultant $\mathrm{BdG}$ equations are solved in Sec. III, where first we tabulate the numerically obtained mean-field ground states, paying a special attention to the striped phases in the dimer-Bose-Einstein-condensate (BEC) limit, and then construct the thermodynamic phase diagrams. The effects of Hartree shifts on the possible ground states are discussed in Sec. V in the context of harmonically confined atomic systems. We end the paper with a brief summary of our conclusions and an outlook in Sec. VI, and an Appendix comparing the dimer-BEC limit in the Landau and symmetric gauges.

\section{THEORETICAL FRAMEWORK}

To explore the ground states of the single-band HofstadterHubbard model, we start with

$$
H=-\sum_{i j \sigma} t_{i j \sigma} a_{i \sigma}^{\dagger} a_{j \sigma}-\sum_{i \sigma} \mu_{i \sigma} a_{i \sigma}^{\dagger} a_{i \sigma}-g \sum_{i} a_{i \uparrow}^{\dagger} a_{i \uparrow} a_{i \downarrow}^{\dagger} a_{i \downarrow},
$$

and consider both thermodynamic and confined systems. Here, $a_{i \sigma}^{\dagger}\left(a_{i \sigma}\right)$ creates (annihilates) a $\sigma \equiv\{\uparrow, \downarrow\}$ fermion on site $i, t_{i j \sigma}$ is its hopping parameter from site $i$ to $j$, and $\mu_{i \uparrow}=$ $\mu-V_{i}+h$ and $\mu_{i \downarrow}=\mu-V_{i}-h$ are effectively their local 
chemical potentials in the presence of confining potential $V_{i}$ and an out-of-plane Zeeman field $h$. We assume $h \geqslant 0$ without losing generality, since $h<0$ results can be easily deduced by letting $\uparrow \rightarrow \downarrow$ and $\downarrow \rightarrow \uparrow$. The density-density interaction term is taken to be local (on site) and attractive with strength $g \geqslant 0$, and the resultant many-body phases are investigated within the mean-field approximation for the Cooper pairs and their superfluidity, as described below.

\section{A. Mean-field Hofstadter-Hubbard Hamiltonian}

In particular, we analyze the following mean-field Hamiltonian for square lattices:

$$
\begin{aligned}
H_{m f}= & -\sum_{i j \sigma} t_{i j \sigma} a_{i \sigma}^{\dagger} a_{j \sigma}-\sum_{i \sigma} \tilde{\mu}_{i \sigma} a_{i \sigma}^{\dagger} a_{i \sigma} \\
& +\sum_{i}\left(\Delta_{i} a_{i \uparrow}^{\dagger} a_{i \downarrow}^{\dagger}+\Delta_{i}^{*} a_{i \downarrow} a_{i \uparrow}+\frac{\left|\Delta_{i}\right|^{2}}{g}\right),
\end{aligned}
$$

where $\tilde{\mu}_{i \uparrow}=\mu_{i \uparrow}-g n_{i \downarrow}$ and $\tilde{\mu}_{i \downarrow}=\mu_{i \downarrow}-g n_{i \uparrow}$ take the Hartree shifts into account. Here, $n_{i \sigma}=\left\langle a_{i \sigma}^{\dagger} a_{i \sigma}\right\rangle$ is the average number of $\sigma$ fermions where $\langle\cdots\rangle$ is a thermal average, and the remaining terms in Eq. (2) involve the complex SF order parameter $\Delta_{i}=g\left\langle a_{i \uparrow} a_{i \downarrow}\right\rangle$. These average quantities are specified below in Eqs. (7)-(9), and we use them in Sec. III for characterizing the state of the system.

The complex hopping parameters are assumed to connect only the nearest-neighbor sites, i.e., $t_{i j \sigma}=t_{\sigma} e^{i \theta_{i j \sigma}}$, where the amplitudes $t_{\uparrow}=t_{\downarrow}=t \geqslant 0$ are taken to be equal for $i$ and $j$ nearest neighbors and 0 otherwise. The phase, however, depends on the external magnetic (or artificial gauge) field experienced by $\sigma$ fermions. In particular, we use the Peierls substitution and take $\theta_{i j \sigma}=\left(1 / \phi_{0}\right) \int_{\mathbf{r}_{i}}^{\mathbf{r}_{j}} \mathbf{A}_{\sigma}(\mathbf{r}) \cdot d \mathbf{r}$, with $\phi_{0}=$ $2 \pi \hbar / e$ the magnetic flux quantum and $\mathbf{A}_{\sigma}(\mathbf{r})$ the vector potential which is assumed to be independently controllable for $\uparrow$ and $\downarrow$ fermions. Note that, while independent control of $\mathbf{A}_{\sigma}(\mathbf{r})$ is not possible for conventional solid-state materials with real magnetic fields where $\sigma$ corresponds to the \pm projections of spin angular momentum of electrons, such a control can be achieved with neutral atomic systems under the influence of laser-generated artificial gauge fields where the pseudospin $\sigma$ is just a label for two of the hyperfine states of a particular atom. In this paper, we choose the Landau gauge for the vector potential, i.e., $\mathbf{A}_{\sigma}(\mathbf{r}) \equiv\left(0, B_{\sigma} x, 0\right)$, leading to a uniform magnetic flux $\Phi_{\sigma}=B_{\sigma} \ell^{2}$ per unit cell penetrating our square lattice, where $\ell$ is the lattice spacing. Denoting the $(x, y)$ coordinates of site $i$ by $(n \ell, m \ell)$, this gauge simply implies $\theta_{i j \sigma}=0$ and $\theta_{i j \sigma}= \pm 2 \pi n \phi_{\sigma}$ for links along the $x$ and $y$ directions, respectively, where $\phi_{\sigma}=\Phi_{\sigma} /\left(2 \pi \phi_{0}\right)$ characterizes the competition between $\ell$ and the magnetic length scale (cyclotron radius) $\ell_{B_{\sigma}}=\sqrt{\hbar /\left(e B_{\sigma}\right)}$. We note that, while $\phi_{\uparrow}=\phi_{\downarrow} \ll 1$ for typical electronic crystals, even for the largest magnetic field $B_{\uparrow}=B_{\downarrow} \sim 100 \mathrm{~T}$ that is attainable in a laboratory, $\phi_{\uparrow}$ and $\phi_{\downarrow}$ may be tuned at will in atomic optical lattices.

Let us first set $g=0$ and $\mu_{i \sigma}=0$ in Eq. (2), and review the well-known single-particle problem, i.e., the Hofstadter Hamiltonian for a uniform square lattice.

\section{B. Hofstadter butterfly}

In the noninteracting limit, the single-particle Hofstadter Hamiltonian describing a $\sigma$ fermion can be written as

$H_{0 \sigma}=-t_{\sigma} \sum_{n m}\left(a_{n m \sigma}^{\dagger} a_{n+1, m \sigma}+e^{i 2 \pi \phi_{\sigma} n} a_{n m \sigma}^{\dagger} a_{n, m+1, \sigma}+\right.$ H.c. $)$,

where H.c. is the Hermitian conjugate. For rational values of $\phi_{\sigma} \equiv p_{\sigma} / q_{\sigma}$, where $p_{\sigma}$ and $q_{\sigma}$ are positive integers with no common factor, i.e., coprime numbers, while $H_{0 \sigma}$ maintains its translational invariance in the $y$ direction, it requires $q_{\sigma}$ sites for translational invariance in the $x$ direction. Thanks to the Bloch theorem, the first magnetic Brillouin zone is determined by $-\pi \leqslant k_{y} \ell \leqslant \pi$ and $-\pi / q_{\sigma} \leqslant k_{x} \ell \leqslant \pi / q_{\sigma}$, and this increased periodicity motivates us to work with a supercell of $1 \times q_{\sigma}$ sites. The excitation spectrum is determined by solving the Schrödinger equation $H_{0 \sigma} \Psi_{\sigma}=\varepsilon\left(\phi_{\sigma}\right) \Psi_{\sigma}$ for all momentum $\mathbf{k} \equiv\left(k_{x}, k_{y}\right)$ values in the first magnetic Brillouin zone. Denoting the components of the wave function as $\Psi_{\sigma}=\left(\psi_{1}^{*}, \psi_{2}^{*}, \psi_{3}^{*}, \ldots, \psi_{q_{\sigma}}^{*}\right)^{\dagger}$, where $\psi_{n}$ corresponds to the $n$th site of the supercell, the $q_{\sigma} \times q_{\sigma}$ Hamiltonian matrix at a given $\mathbf{k}$ value

$$
\left[\begin{array}{ccccccc}
C_{1 \sigma} & T_{\sigma}^{*} & 0 & . & . & 0 & T_{\sigma} \\
T_{\sigma} & C_{2 \sigma} & T_{\sigma}^{*} & 0 & \cdot & \cdot & 0 \\
0 & T_{\sigma} & C_{3 \sigma} & \cdot & \cdot & . & \cdot \\
\cdot & \cdot & \cdot & \cdot & \cdot & \cdot & 0 \\
0 & \cdot & \cdot & \cdot & \cdot & C_{n-1, \sigma} & T_{\sigma}^{*} \\
T_{\sigma}^{*} & 0 & \cdot & \cdot & 0 & T_{\sigma} & C_{n \sigma}
\end{array}\right]
$$

describes the supercell with periodic Bloch boundary conditions. Here, $C_{n \sigma}=-2 t_{\sigma} \cos \left(k_{y} \ell+2 \pi n p_{\sigma} / q_{\sigma}\right)$ and $T_{\sigma}=$ $-t_{\sigma} e^{i k_{x} \ell}$.

The eigenvalues $\varepsilon\left(\phi_{\sigma}\right)$ of this $q_{\sigma} \times q_{\sigma}$ matrix can be numerically obtained for any given rational number $\phi_{\sigma}$ and the energy spectrum $\varepsilon\left(\phi_{\sigma}\right)$ vs $\phi$ is known as the Hofstadter butterfly $(\mathrm{HB})[1,2]$. The spectrum is shown in Fig. 1, where,

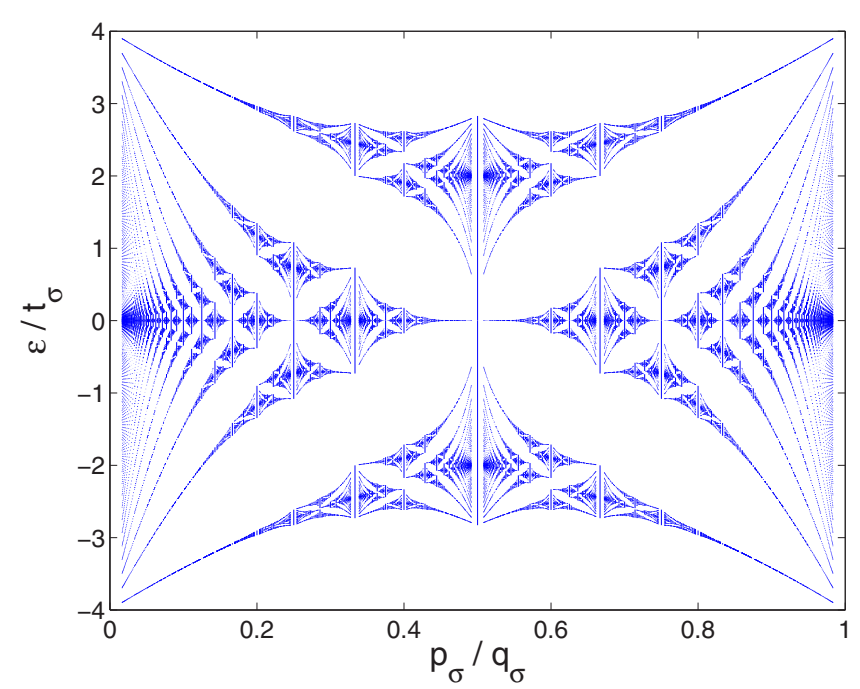

FIG. 1. (Color online) The Hofstadter spectrum $\varepsilon / t_{\sigma}$ as a function of $\phi_{\sigma}=p_{\sigma} / q_{\sigma}$, showing its fractal structure with numerous sub-bands and minigaps. 
for a given $\phi_{\sigma}$, it consists of nonoverlapping $q_{\sigma}$ bands with $q_{\sigma}+1$ energy gaps in between, and each one of these $q_{\sigma}$ bands can accommodate a filling of $1 / q_{\sigma}$ particles with a total filling of 1 . Therefore, if we index the energy gaps as $z_{\sigma}=\left\{0,1,2, \ldots, q_{\sigma}\right\}$, starting from the bottom edge of the band in such a way that the lowest $\left(z_{\sigma}=0\right)$ and highest ( $\left.z_{\sigma}=q_{\sigma}\right)$ gaps correspond, respectively, to a particle vacuum and a fully filled band insulator, particle fillings within all of these gapped regions can be compactly written as $z_{\sigma} / q_{\sigma}$. Note that while all gaps are open for odd $q_{\sigma}$, the middle $z_{\sigma}=q_{\sigma} / 2$ gap corresponding to a half-filled lattice is not open when $q_{\sigma}$ is even, and therefore a half-filled lattice is not an insulator for any $q_{\sigma}$. In the $\phi_{\sigma} \rightarrow 0$ limit, the HB spectrum recovers the usual tight-binding dispersion of cosines, $\varepsilon_{\mathbf{k} \sigma}=$ $-2 t_{\sigma}\left[\cos \left(k_{x} \ell\right)+\cos \left(k_{y} \ell\right)\right]$, which has an energy bandwidth $W_{\sigma}=8 t_{\sigma}$.

Since $\ell$ and $\ell_{B_{\sigma}}$ are the only two length scales in Eq. (3) such that $\phi_{\sigma}=\ell^{2} /\left(2 \pi \ell_{B_{\sigma}}^{2}\right)$, the fractal structure of the HB is clearly a result of their competition. In addition, each $\mathbf{k}$ state is $q_{\sigma}$-fold degenerate in the first magnetic Brillouin zone (not explicitly shown in the figure), i.e.,

$$
\varepsilon_{b, k_{x} k_{y}}\left(\phi_{\sigma}\right)=\varepsilon_{b, k_{x}, k_{y}+2 \pi \phi_{\sigma} f / \ell}\left(\phi_{\sigma}\right)
$$

with $b=1,2, \ldots, q_{\sigma}$ labeling the bands and $f=1,2, \ldots, q_{\sigma}$ labeling the degenerate $\mathbf{k}$ states. We have recently shown that the $\mathrm{HB}$ spectrum plays a crucial role in determining the many-body states of the interacting system [23], and our primary objective here is to extend and generalize the analysis to imbalanced gauge fields.

\section{Bogoliubov-de Gennes theory}

For this purpose, we diagonalize Eq. (2) via the BogoliubovValatin transformation, i.e., $a_{i \sigma}=\sum_{m}\left(u_{m i \sigma} \gamma_{m \sigma}-\right.$ $\left.s_{\sigma} v_{m i \sigma}^{*} \gamma_{m,-\sigma}^{\dagger}\right)$, where $\gamma_{m \sigma}^{\dagger}\left(\gamma_{m \sigma}\right)$ creates (annihilates) a pseudospin- $\sigma$ quasiparticle with energy $\epsilon_{m}^{\sigma}$ and wave functions $u_{m i \sigma}$ and $v_{m i \sigma}$, and $s_{\uparrow}=+1$ and $s_{\downarrow}=-1$. The resultant $\mathrm{BdG}$ equations can be compactly written as

$$
\sum_{j}\left(\begin{array}{cc}
-t_{i j \uparrow}-\tilde{\mu}_{i \uparrow} \delta_{i j} & \Delta_{i} \delta_{i j} \\
\Delta_{i}^{*} \delta_{i j} & t_{i j \downarrow}^{*}+\tilde{\mu}_{i \downarrow} \delta_{i j}
\end{array}\right) \varphi_{m j}^{\sigma}=s_{\sigma} \epsilon_{m}^{\sigma} \varphi_{m i}^{\sigma},
$$

where $\delta_{i j}$ is the Kronecker delta, and $\varphi_{m i}^{\uparrow}=\left(u_{m i \uparrow}^{*}, v_{m i \downarrow}^{*}\right)^{\dagger}$ and $\varphi_{m i}^{\downarrow}=\left(v_{m i \uparrow},-u_{m i \downarrow}\right)^{\dagger}$ are the corresponding eigenfunctions for the eigenvalue $\epsilon_{m}^{\sigma} \geqslant 0$. Note that the $\mathrm{BdG}$ equations are invariant under the transformation $v_{m i \uparrow} \rightarrow u_{m i \uparrow}^{*}, u_{m i \downarrow} \rightarrow$ $-v_{m i \downarrow}^{*}$, and $\epsilon_{m \downarrow} \rightarrow-\epsilon_{m \uparrow}$, and therefore it is sufficient to solve only for $u_{m i} \equiv u_{m i \uparrow}, v_{m i} \equiv v_{m i \downarrow}$, and $\epsilon_{m} \equiv \epsilon_{m}^{\uparrow}$ as long as all solutions with positive and negative $\epsilon_{m}$ are kept.

Using the transformation, the complex order parameter $\Delta_{i}$ can be written as

$$
\Delta_{i}=-g \sum_{m} u_{m i} v_{m i}^{*} f\left(\epsilon_{m}\right),
$$

where $f(x)=1 /\left[e^{x /\left(k_{B} T\right)}+1\right]$ is the Fermi function with $k_{B}$ the Boltzmann constant and $T$ the temperature. Equations (6) and (7) have to be solved self-consistently for a given $\mu$ and $h$, such that the total number of $\sigma$ fermions satisfies $N_{\sigma}=$ $\sum_{i} n_{i \sigma}$. Here, $0 \leqslant n_{i \sigma}=\left\langle a_{i \sigma}^{\dagger} a_{i \sigma}\right\rangle \leqslant 1$ is the average number of $\sigma$ fermions on site $i$, and using the transformation, it can be written as

$$
\begin{aligned}
& n_{i \uparrow}=\sum_{m}\left|u_{m i}\right|^{2} f\left(\epsilon_{m}\right), \\
& n_{i \downarrow}=\sum_{m}\left|v_{m i}\right|^{2} f\left(-\epsilon_{m}\right)
\end{aligned}
$$

for the $\uparrow$ and $\downarrow$ fermions, respectively. We note that unlike the continuum models where the solutions of the self-consistency equations depend explicitly on the high-momentum cutoff, requiring a high-energy regularization in order to obtain cutoffindependent results, the lattice versions given in Eqs. (7)-(9) do not require such a regularization, since the lattice spacing $\ell$ already provides an implicit short-distance cutoff.

In the absence of gauge fields when $\theta_{i j}=0$, it is generally accepted that the mean-field description given above provides qualitative understanding either at low temperatures $(T \ll$ $T_{c}$ ) for any $g$ or for weak $g \lesssim W$ at any $T$, where $T_{c}$ is the critical SF transition temperature. It is also known that single-band Hubbard models gradually become inadequate in describing strongly interacting cold-atom systems on optical lattices, requiring multiband models [24]. In addition, the real-space $\mathrm{BdG}$ theory goes beyond the standard local-density approximation since it includes both $\theta_{i j}$ and $V_{i}$ exactly into the mean-field theory without relying on further approximations. Hoping to shed light on the qualitative effects of gauge fields on the ground states of Eq. (2), here we mainly concentrate on weak and intermediate $g$ at $T=0$ as discussed next.

\section{NUMERICAL FRAMEWORK}

In order to explore the possible phases, let us set $V_{i}=0$ and consider a uniform $45 \ell \times 45 \ell$ square lattice, which is large enough to construct the thermodynamic phase diagrams for $\phi_{\sigma}=\{0,1 / 6, \pm 1 / 4\}$. We note that, even though our phase diagrams are reliable, the phase boundaries should be taken as qualitative guides to the eye due to the possibility of minor finite-size effects. We neglect the Hartree shifts for the moment because not only do the self-consistent solutions converge much faster but also the resultant phase diagrams are much easier to interpret and understand. In addition, since none of the PDW, CDW, and SDW instabilities are driven by these shifts, our qualitative mean-field results already pave the way to quantitative understanding of the possible ground states of Eq. (2). However, see Sec. V B for the effects of Hartree shifts on confined systems.

For this purpose, we numerically solve Eqs. (6)-(9) at $T=0$ and obtain self-consistent solutions of $\Delta_{i} / t$ and $n_{i \sigma}$ as functions of $g / t, \mu / t, h / t$, and $\phi_{\sigma}$. This can be achieved numerically via the iterative method of relaxation as follows. For a given set of parameters, first (i) start with an input set of $\Delta_{i}$, then (ii) construct the BdG matrix given in Eq. (6), and then (iii) use its eigenstates in Eq. (7) to generate a new set of $\Delta_{i}$, and finally (iv) repeat these steps until the input and output sets of $\Delta_{i}$ lie within a confidence level. Once this iterative method converges, (v) use Eqs. (8) and (9) to calculate $n_{i \sigma}$. It turns out that while Eqs. (6)-(9) have unique solutions in the low- $(h / g)$ limit, they in general allow for multiple solutions for the polarized many-body phases, and therefore it is essential 
TABLE I. While the SSS* phase has a small but finite signchanging striped-SDW order, the system is globally unpolarized, very much like the unpolarized uniform superfluid (USF) or unpolarized striped supersolid (SSS) phase. PSF denotes the polarized superfluid phase.

\begin{tabular}{lcccc}
\hline \hline Phase & $\left|\Delta_{i}\right|$ & $n_{i \uparrow}+n_{i \downarrow}$ & $n_{i \uparrow}-n_{i \downarrow}$ & $\phi_{\sigma}$ \\
\hline USF & Uniform & Uniform & 0 & $\phi_{\uparrow}=-\phi_{\downarrow}$ \\
SSF & PDW & 1 & 0 & $\phi_{\uparrow}=\phi_{\downarrow}$ \\
SSS & PDW & CDW & 0 & $\phi_{\uparrow}=\phi_{\downarrow}$ \\
SSS* & PDW & CDW & SDW & $\left|\phi_{\uparrow}\right| \neq\left|\phi_{\downarrow}\right|$ \\
PSF & \multicolumn{4}{c}{ otherwise } \\
\hline \hline
\end{tabular}

to try several initial sets of $\Delta_{i}$ and verify the (meta)stability of the solutions.

\section{A. Ground-state phases}

Depending on the spatial profiles of $\left|\Delta_{i}\right|, n_{i \uparrow}$, and $n_{i \downarrow}$, we distinguish the single-particle band insulator $(I)$ and normal $(N)$ phases from the ordered many-body ones using the following criteria. When $h / g$ is sufficiently high that $\Delta_{i} \rightarrow 0$ (precisely speaking, $\left|\Delta_{i}\right|<10^{-3} t$ in our numerics) for every $i$, the ground state can be a $\sigma$-vac phase which is a vacuum of $\sigma$ component with $n_{i \sigma}=0$, a $\sigma-I(m / n)$ phase which is a band insulator of $\sigma$ component with uniform $n_{i \sigma}=m / n$, a $\sigma-N$ phase which is a normal $\sigma$ component, or an $\uparrow \downarrow-P N$ phase, which is a polarized normal mixture of $\uparrow$ and $\downarrow$ components. We checked in our numerics that while the $\sigma-N$ and $\uparrow \downarrow-P N$ phases have slightly nonuniform $n_{i \sigma}$ for $\phi \neq 0$, the $C_{4}$ symmetry of the square lattice is preserved. On the other hand, when $h / g$ is sufficiently low that $\Delta_{i} \neq 0$ (i.e., $\left|\Delta_{i}\right|>$ $10^{-3} t$ ) for some $i$, the ground states can be characterized according to Table I. Unlike our earlier work [23], here we do not finely classify the polarized superfluid phase depending on the coexisting (striped or nonstriped) PDW, CDW, SDW, and/or VL orders. Instead, we focus mostly on the existence of striped phases in the dimer-BEC limit as the main message of this paper, for which physical (analytical) insight are also given.

The globally unpolarized states are denoted by USF, SSF, and SSS, and they stand, respectively, for uniform-SF, stripedSF, and striped-SS states. The SSS* state is also globally unpolarized, very much like the SSS phase but it has an additional sign-changing striped-SDW order driven by the imbalance between $\phi_{\uparrow}$ and $\phi_{\downarrow}$. For instance, typical $\left|\Delta_{i}\right|$ and $n_{i \uparrow} \pm n_{i \downarrow}$ profiles are illustrated in Fig. 2 for all of them. Depending on $\mu, h, \phi_{\uparrow}$, and $\phi_{\downarrow}$, one of the USF, SSF, SSS, and SSS* phases always appears in the thermodynamic phase diagrams beyond a critical $g / t$ threshold, as discussed next.

\section{B. Dimer-BEC limit in the Landau gauge}

When $g / t \gg 1$ is sufficiently high, the physics must eventually be determined by the two-body bound states, i.e., Cooper pairs become bosonic dimers, and unless $g / t \rightarrow \infty$, the dimer-dimer interaction $\left[g_{d d} \sim\left(t_{\uparrow}^{2}+t_{\downarrow}^{2}\right) / g\right]$ is finite. Such weakly repulsive dimers can effectively be described by the Hofstadter-Bose-Hubbard model, where superfluidity has
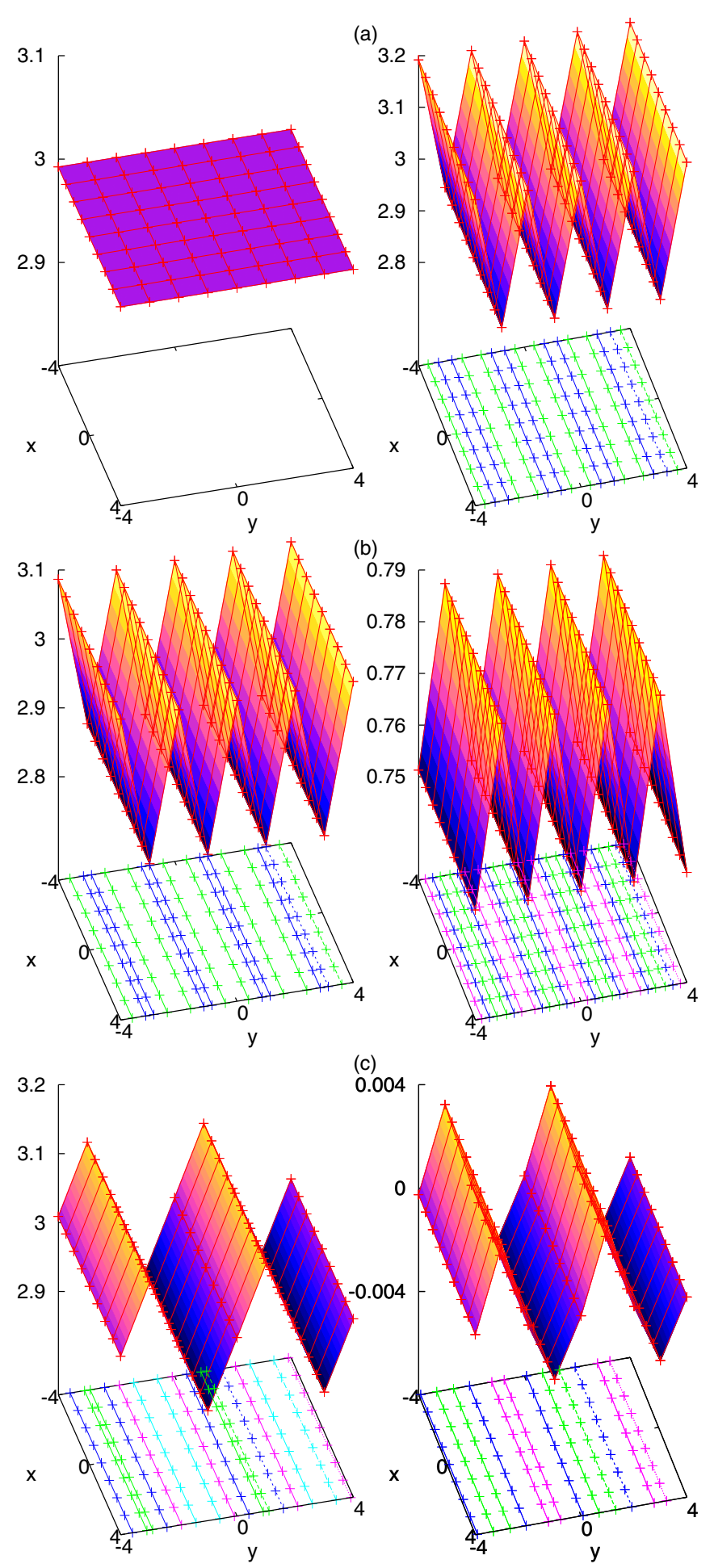

FIG. 2. (Color online) Characterization of globally unpolarized many-body phases. (a) Typical $\left|\Delta_{i}\right| / t$ profiles are shown for the USF (left) and SSF phases (right), where $\phi_{\uparrow}=-\phi_{\downarrow}=1 / 4$ and $\phi_{\uparrow}=\phi_{\downarrow}=$ $1 / 4$, respectively, and $\mu=0$ (uniformly half filled) in both figures. (b) Typical $\left|\Delta_{i}\right| / t$ (left) and $n_{i \uparrow}+n_{i \downarrow}$ (right) profiles are shown for the SSS phase, where $\phi_{\uparrow}=\phi_{\downarrow}=1 / 4$ and $\mu=-t$. (c) Typical $\left|\Delta_{i}\right| / t$ and $n_{i \uparrow}-n_{i \downarrow}$ profiles are shown for the SSS* phase, where $\phi_{\uparrow}=0$, $\phi_{\downarrow}=1 / 4$, and $\mu=-t$. Note in (c) that even though the system is globally unpolarized, it has both SDW and CDW (not shown) orders. Here, $(x, y)$ are in units of $\ell$, and we set $h=0$ and $g=7 t$ in all figures. 
recently been shown to break translation symmetry in the weakly interacting limit [25].

In the ideal-dimer limit of our model Hamiltonian, the only way for a tightly bound dimer to move from a site $i$ to $j$ in the lattice is via what is known as the pair-breaking mechanism, i.e., virtual ionization of its constituents costs a penalty of $g$, and this gives rise to the effective dimer hopping parameter $t_{i j d}=2 t_{i j \uparrow} t_{i j \downarrow} / g$. Therefore, the effective hopping amplitude and gauge field of the dimers can be written as $t_{d} \approx 2 t_{\uparrow} t_{\downarrow} / g$ and $\phi_{d}=\phi_{\uparrow}+\phi_{\downarrow}=p_{d} / q_{d}$, respectively, where $p_{d}=\left(p_{\uparrow} q_{\downarrow}+p_{\downarrow} q_{\uparrow}\right) / Q$ and $q_{d}=q_{\uparrow} q_{\downarrow} / Q$. Here, $Q$ is a positive integer number chosen such that $p_{d}$ and $q_{d}$ are coprime numbers, and it depends on the entire $\left\{p_{\uparrow}, p_{\downarrow}, q_{\uparrow}, q_{\downarrow}\right\}$ set. Since the HB for dimers is $q_{d}$-fold degenerate, the BEC order parameter has contributions from all degenerate $\mathbf{k}_{d}=\left\{(0,0) ;\left(0,2 \pi \phi_{d} f / \ell\right)\right\}$ momenta, where $f=1, \ldots, q_{d}-$ 1 such that $\Psi_{i d}=c_{0}+\sum_{f} c_{f} e^{i 2 \pi \phi_{d} f i_{y} / \ell}$ and $c_{f}=\left|c_{f}\right| e^{i \vartheta_{f}}$ are complex variational parameters. However, unlike atomic bosons where all of the degenerate states have equal weights, dimer bosons are fermion pairs, and the number of ways of creating them with $k_{y d}=k_{y \uparrow}+k_{y \downarrow}$ momentum depends on $f, \phi_{\uparrow}$, and $\phi_{\downarrow}$. For instance, there are $2(q-f)-1$ ways of intraband pairing when $\phi_{\uparrow}=\phi_{\downarrow}=p / q$ and $q$ is even. Thus, this analysis show that higher- $k_{y d}$ states contribute less and less, forming a perturbative series.

It turns out that the first-order $(f=1)$ correction is already much smaller than the zeroth-order $(f=0)$ one, and that the $f \geqslant 2$ terms are always negligible when $g / t$ is sufficiently large. This is because all of our numerical results fit quite well with

$$
\left|\Delta_{i}\right|=\left|\Delta_{0}\right|+\left|\Delta_{1}\right|\left[1-\cos \left(2 \pi \phi_{d} i_{y} / \ell\right)\right]
$$

in the entire globally unpolarized region, including SSF, SSS, and SSS* phases. Here, the $\mathbf{k}_{d}=(0,0)$ contribution $\left|\Delta_{0}\right|=$ $\left(g / 2-4 t^{2} / g\right) \sqrt{n(2-n)}$ is uniform in space and determined by the total average filling $n$ with $\mu=\left(g / 2-8 t^{2} / g\right)(n-$ 1) [26], $\left|\Delta_{1}\right| \approx t^{2} / g$ for $\mu \approx 0$, and $i_{y}$ is the $y$ coordinate of site $i$. Moving towards the BCS side, the second-order correction to Eq. (10) can be shown to be $+\left|\Delta_{2}\right| \cos \left(4 \pi \phi_{d} i_{y} / \ell\right)$ for even $q_{d}$. Since this term is in ( $\pi$ out of ) phase with the zeroth- (first-) order term, it tends to open troughs along the peaks arisising from the first-order one, suggesting that $\vartheta_{f}-\vartheta_{0}=\pi f$, i.e., the form of $\left|\Psi_{i d}\right|$ coincides with $\left|\Delta_{i}\right|$ under these conditions. Equation (10) clearly shows that modulations of $\left|\Delta_{i}\right|$ have a spatial period of $q_{d}$ lattice sites along the $y$ direction. It also implies that it is the cooperation between $\phi_{d}$ and $g$ that is responsible for the broken spatial symmetry and appearance of stripe order, and even though the stripe order gradually fades away with increasing $g$, it survives even in the $g \gg W$ limit as long as $g / t$ is finite.

Thus, this analysis suggests that the existence of stripeordered SF and SS phases is not an artifact of the mean-field description, and they are physically expected in the dimer-BEC limit of the attractive Hofstadter-Hubbard model, as discussed next.

\section{THERMODYNAMIC PHASE DIAGRAMS}

Despite tremendous efforts over several decades, the exact phase diagram of even the simplest Hubbard model (which

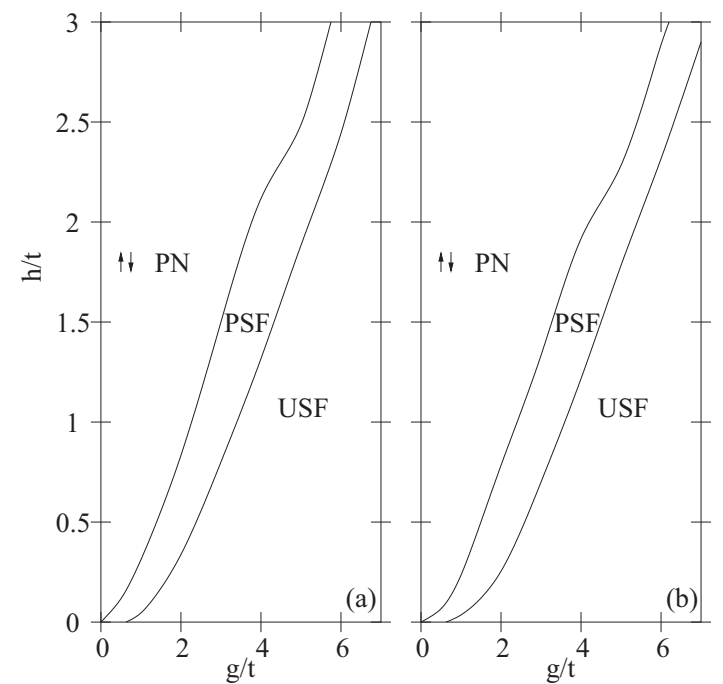

FIG. 3. No gauge field: the $\phi_{\uparrow}=\phi_{\downarrow}=0$ case. The ground-state phase diagrams are shown for $\mu=0$ in (a) and $\mu=-t$ in (b).

does not include the gauge fields or Zeeman fields) is still the subject of a hot debate; however, the mean-field phases and resultant phase diagrams of the mean-field Hubbard model are pretty much settled. To appreciate the effects of gauge fields, first we study Eq. (2) with $\phi_{\uparrow}=\phi_{\downarrow}=0$.

\section{A. No gauge fields: $\phi_{\uparrow}=\phi_{\downarrow}=0$}

Our results for this limit are presented in Fig. 3, where we set $\mu=0$ in Fig. 3(a) corresponding to a half-filled lattice, and $\mu=-t$ in Fig. 3(b). We find that the phase diagrams are very similar, and depending on the particular value of $g$, there are two critical $h$ fields. Since the (FFLO) phase occupies a tiny parameter space near the normal phase boundary and only on the BCS side when $g / t \lesssim W$, we do not finely classify the character of the PSF phase in Fig. 3 and throughout this paper. The USF phase, where $\Delta_{i}=\Delta_{0}$ for all $i$, turns into a PSF phase beyond a first critical field $h_{c_{1}}$, and then the PSF phase becomes an $\uparrow \downarrow-P N$ phase beyond a second critical field $h_{c_{2}}>h_{c_{1}}$. Our numerical results indicate that $h_{c_{1}} \sim\left|\Delta_{0}\right|$ where $\left|\Delta_{0}\right|$ is evaluated at $h=0$ for the same parameters.

In the strongly interacting limit when $g \gg t$, it can be analytically shown for thermodynamic systems that $\left|\Delta_{0}\right|=$ $\left(g / 2-4 t^{2} / g\right) \sqrt{n(2-n)}$, where $n=n_{\uparrow}+n_{\downarrow}$ is the total fermion filling. We checked that this thermodynamic expression agrees very well with our finite-lattice results, as it gives $\left|\Delta_{0}\right| \approx 7.23 t$ for $\mu=0$ or $n=1$ and $\left|\Delta_{0}\right| \approx 7.18 t$ for $\mu=-t$ or $n \approx 0.875$ when $g=15 t$, while we find, respectively, $\left|\Delta_{0}\right| \approx 7.25 t$ and $\left|\Delta_{0}\right| \approx 7.19 t$ for the same parameters in our BdG calculations. In the weakly interacting limit when $g$ is sufficiently small so that $\Delta_{i} \rightarrow 0$ for every $i$, we note that the system will be a $\downarrow$-vac system for $h>4 t$ when $\mu=0$ and for $h>3 t$ when $\mu=-t$. Next, we are ready to discuss the effects of balanced gauge fields.

\section{B. Balanced gauge fields: $\phi_{\uparrow}=\phi_{\downarrow} \neq 0$}

In Fig. 4, we present the $\phi_{\sigma}=1 / 4$ phase diagrams for $\mu=0$ in Fig. 4(a) and $\mu=-t$ in Fig. 4(b). The $\mu=0$ case 

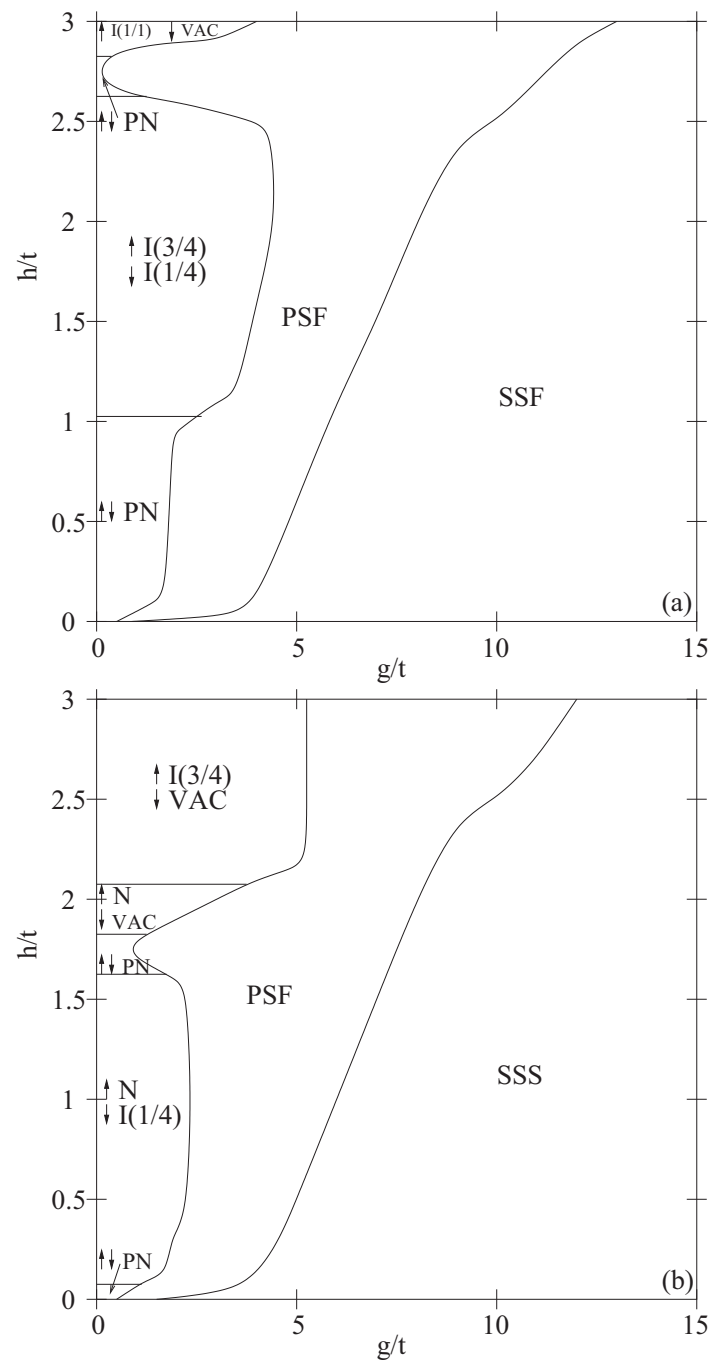

FIG. 4. $\phi_{\uparrow}=\phi_{\downarrow}=1 / 4$ case. The ground-state phase diagrams are shown for $\mu=0$ in (a) and $\mu=-t$ in (b).

is very special since it corresponds to a half-filled lattice with particle-hole symmetry, where $n_{i \uparrow}+n_{i \downarrow}=1$ independently of $i$, no matter what the rest of the parameters are. In comparison to Fig. 3 , the $\phi_{\sigma}=1 / 4$ diagrams have much richer structure involving large regions of stripe-ordered phases. To understand the physical origin of the resultant phase diagrams and stripe order, next we discuss the analytically tractable high- and low$(h / g)$ limits.

When $h / g$ is sufficiently high, we can directly read off the single-particle ground state of the $\sigma$ component from the $\mathrm{HB}$ for any given $\phi_{\sigma}$. For $\phi_{\sigma}=1 / 4$, the energy spectrum consists of four bands: the $\sigma$ component is a $\sigma$-vac for $\mu_{\sigma} \lesssim-2.83 t$, a $\sigma-N$ for $-2.83 t \lesssim \mu_{\sigma} \lesssim-2.61 t$, a $\sigma-I(1 / 4)$ for $-2.61 t \lesssim \mu_{\sigma} \lesssim-1.082 t$, a $\sigma-N$ for $-1.082 t \lesssim \mu_{\sigma} \lesssim$ $1.082 t$, a $\sigma-I(3 / 4)$ for $1.082 t \lesssim \mu_{\sigma} \lesssim 2.61 t$, a $\sigma-N$ for $2.61 t \lesssim \mu_{\sigma} \lesssim 2.83 t$, and a $\sigma-I(1 / 1)$ for $2.83 t \lesssim \mu_{\sigma}$. Using $\mu_{\uparrow}=\mu+h$ and $\mu_{\downarrow}=\mu-h$ in these expressions, the high$(h / g)$ structure of Fig. 4 immediately follows. As $h / g$ gets smaller, the single-particle $I$ and $N$ phases must pave the way to ordered many-body ones, as increasing the strength of the pairing (attractive potential) energy eventually makes them energetically less favorable. For $\phi_{\sigma}=0$, it is intuitively expected and numerically confirmed above that the $\uparrow \downarrow-P N$ to the PSF phase transition boundary $g\left(h_{c}\right)$ is a monotonic function of $h$, which is simply because the noninteracting system has a very simple band structure with cosine dispersions. However, due to the presence of multiple bands, the transition boundary $g\left(h_{c}\right)$ becomes a complicated function of $h$ for finite $\phi_{\sigma}$. For instance, we find a sizable hump in Fig. 4(a) around $h \approx 2.7 t$ and another one in Fig. 4(b) around $h \approx 1.7 t$, the peak locations of which coincide intuitively with the $\uparrow \downarrow-P N$ regions that are sandwiched between vac and/or $I$.

On the other hand, when $h / g$ is sufficiently small, the ground state is expected to be an ordered many-body phase with no polarization. In sharp contrast to the $\phi_{\sigma}=0$ case where USF is numerically confirmed above to be the ground state for any $\mu$, we show in Fig. 4 that the SSF and SSS phases are, respectively, stable for $\mu=0$ and $\mu=-t$ when $\phi_{\sigma}=1 / 4$. Note that since $\mu=0$ corresponds to half filling for any $\phi_{\sigma}$, the unpolarized ground states necessarily have uniform fillings, i.e., $n_{i \uparrow}=n_{i \downarrow}=1 / 2$ for every $i$. Therefore, in the low- $(h / g)$ limit, while only $\left|\Delta_{i}\right|$ is allowed to have spatial modulations in Fig. 4(a), both $\left|\Delta_{i}\right|$ and $n_{i \sigma}$ are modulated in Fig. 4(b).

In comparison, the $\phi_{\sigma}=1 / 6$ phase diagrams are shown in Fig. 5, and they are in many ways similar to the $\phi_{\sigma}=1 / 4$ ones. The main difference is in the high- $(h / g)$ limit which again directly follows from the HB. For $\phi_{\sigma}=1 / 6$, the energy spectrum consists of six bands: the $\sigma$ component is a $\sigma$-vac state for $\mu_{\sigma} \lesssim-3.076 t$, a $\sigma-N$ for a narrow band around $\mu_{\sigma} \approx-3.076 t$, a $\sigma-I(1 / 6)$ for $-3.076 t \lesssim \mu_{\sigma} \lesssim-1.59 t$, a $\sigma-N$ for $-1.59 t \lesssim \mu_{\sigma} \lesssim-1.41 t$, a $\sigma-I(1 / 3)$ for $-1.41 t \lesssim$ $\mu_{\sigma} \lesssim-0.65 t$, a $\sigma-N$ for $-0.65 t \lesssim \mu_{\sigma} \lesssim 0.65 t$, a $\sigma-I(2 / 3)$ for $0.65 t \lesssim \mu_{\sigma} \lesssim 1.41 t$, a $\sigma-N$ for $1.41 t \lesssim \mu_{\sigma} \lesssim 1.59 t$, a $\sigma-I(5 / 6)$ for $1.59 t \lesssim \mu_{\sigma} \lesssim 3.076 t$, a $\sigma-N$ for a narrow band around $\mu_{\sigma} \approx 3.076 t$, and a $\sigma-I(1 / 1)$ for $3.076 t \lesssim \mu_{\sigma}$. As a consequence of this, we note in Fig. 5(b) that the system intuitively requires a finite threshold for $g / t$ even at $h=0$, in order to develop any kind of many-body order. In addition, it is intriguing to see that the sliver of $\downarrow-N$ region that is sandwiched between $\downarrow$-vac and $\downarrow-I(1 / 6)$ around $h \approx 2.076 t$ gives rise to a sizable hump in Fig. 5(b). This is clearly a result of increased single-particle density of states.

Note in Figs. 3-5 that the transition from an unpolarized to a polarized ordered phase occurs at a lower $h$ for any given $g$ as $\phi_{\sigma}$ is increased from 0 . This is a consequence of smaller noninteracting energy bandwidths: as $\phi_{\sigma}$ increases from 0 to $1 / 6$ to $1 / 4$ then $W$ shrinks from $8 t$ to $6.15 t$ to $5.65 t$, making it possible to polarize the ground state with a smaller and smaller $h$. In Figs. 4 and 5, the PSF regions are dominated mainly by a phase that can be characterized by almost striped PDW and SDW orders with some additional corrugations along the stripes that is caused because $h \neq 0$. For instance, when this phase is near to an insulating one, it generally has a very small SDW order in the background on top of a large and uniform polarization.

\section{Imbalanced gauge fields: $\phi_{\uparrow} \neq \phi_{\downarrow}$}

As we argued in Secs. I and II A, while independent control of the gauge fields $\phi_{\uparrow}$ and $\phi_{\downarrow}$ is not possible for conventional solid-state materials with real magnetic fields, such a control 

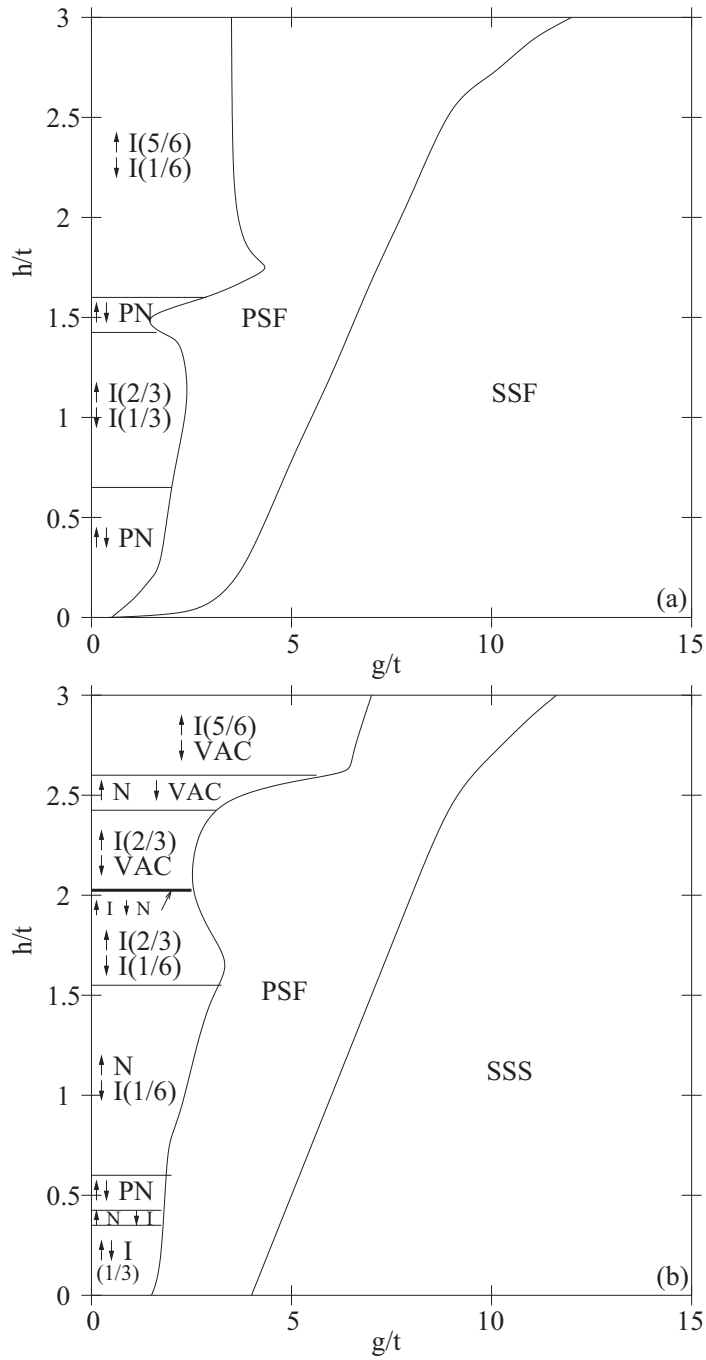

FIG. 5. $\phi_{\uparrow}=\phi_{\downarrow}=1 / 6$ case. The ground-state phase diagrams are shown for $\mu=0$ in (a) and $\mu=-t$ in (b). Note in (b) the presence of a sliver of $\mathrm{a} \downarrow-N$ region around $h \approx 2.076 t$.

is plausible with neutral atomic systems. Motivated by this exotic possibility, here we study two different limits.

As the first limit, we consider a pair of time-reversalsymmetric gauge fields, i.e., $\phi_{\uparrow}=-\phi_{\downarrow}$. For instance, $\phi_{\uparrow}=$ $1 / 4$ phase diagrams are shown in Fig. 6 , where we set $\mu=0$ in Fig. 6(a) and $\mu=-t$ in Fig. 6(b). Thanks to the time-reversal symmetry, even though the ground state is not a PSF but an unpolarized SF phase at $h=0$, it is not properly indicated in these figures for low $g / t$. The general structures of the transition boundaries that are seen in these phase diagrams are quite similar to the ones shown in Fig. 4 for the $\phi_{\uparrow}=\phi_{\downarrow}=1 / 4$ case. However, there is an important caveat in the dimer-BEC limit: the ground state becomes a USF for any $\mu$ as long as $h / g$ is sufficiently low. Given our analysis in Sec. III B, this is intuitively expected since the effective gauge field of Cooper pairs vanishes $\left(\phi_{d}=0\right)$ in the dimer-BEC limit as the gauge fields of $\uparrow$ and $\downarrow$ fermions precisely cancel each other. In addition, the PSF regions necessarily shrink here, since the USF to PSF transition boundaries are expected to be close to the no-gauge-field ( $\phi_{\sigma}=0$ ) ones shown in Fig. 3 .

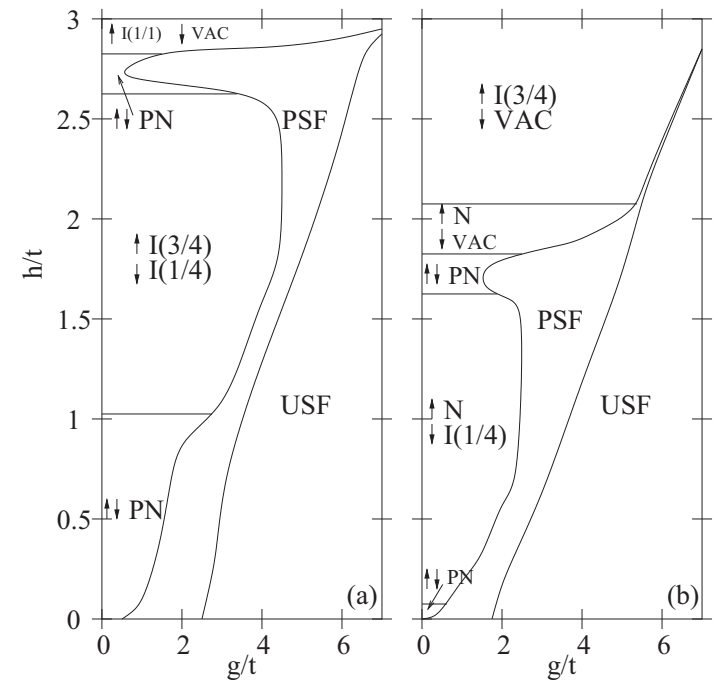

FIG. 6. Time-reversal-symmetric gauge fields: $\phi_{\uparrow}=-\phi_{\downarrow}=1 / 4$ case. The ground-state phase diagrams are shown for $\mu=0$ in (a) and $\mu=-t$ in (b).

As the second limit, we set one of the gauge fields to zero, corresponding effectively to a charged-uncharged mixture of two-component fermions. For instance, the $\left(\phi_{\uparrow}=\right.$ $0, \phi_{\downarrow}=1 / 4$ ) phase diagrams are shown in Fig. 7, where we set $\mu=0$ in Fig. 7(a) and $\mu=-t$ in Fig. 7(b), and the $\left(\phi_{\uparrow}=1 / 4, \phi_{\downarrow}=0\right)$ diagram is shown in Fig. 7(c) where we set $\mu=-t$. Thanks to the particle-hole symmetry around half filling, the $\left(\phi_{\uparrow}=1 / 4, \phi_{\downarrow}=0\right)$ phase diagram for $\mu=0$ can easily be deduced from Fig. 7(a) via $\uparrow \rightarrow \downarrow$ and $\downarrow \rightarrow \uparrow$, and therefore, it is not shown. Since this symmetry also prevents polarization at $h=0$, even though the ground state is not a PSF but an unpolarized nonuniform (but nonstriped) SF for weak $g / t$, this is not properly indicated in Fig. 7(a). However, the imbalance between gauge fields causes a PSF in Figs. 7(b) and 7(c) even at $h=0$. As in the analysis given in Sec. IV B, the high- and low- $(h / g)$ limits can be directly read off from the HB and effective dimer-BEC descriptions, respectively, with again an important caveat in the dimer-BEC limit: the ground state becomes a SSS* for $\mu \neq 0$ as long as $h / g$ is sufficiently low. As shown in Fig. 2(c), in addition to the coexisting striped-PDW and -CDW orders, SSS*has an additional sign-changing striped-SDW order driven solely by $\phi_{\uparrow} \neq \phi_{\downarrow}$. Note also that if $\left(\phi_{\uparrow} \neq 0, \phi_{\downarrow}=0\right)$ then all of the coexisting orders of the SSS* phase are periodic along the $y$ direction with periodicity $q_{d}=q_{\uparrow}$ since $\phi_{d}=\phi_{\uparrow}$.

\section{Stripe order vs FFLO modulations}

It is clearly the cooperation between $g, \phi_{\uparrow}$, and $\phi_{\downarrow}$ that is responsible for the broken spatial symmetry and appearance of stripe order, causing much more prominent stripes for intermediate $g$ at a given $h$. The stripe order is a direct result of the HB: for a given $\phi_{\sigma}$, the spectrum consists of $q_{\sigma}$ bands in the first magnetic Brillouin zone within which each $\mathbf{k}$ state is $q_{\sigma}$-fold degenerate. Therefore, when $g \neq 0$, not only intraand interband pairings but also pairings with both 0 and a set of nonzero center-of-mass momenta are allowed [21,22], leading to a nonuniform $\left|\Delta_{i}\right|$ with spatially periodic modulations, 

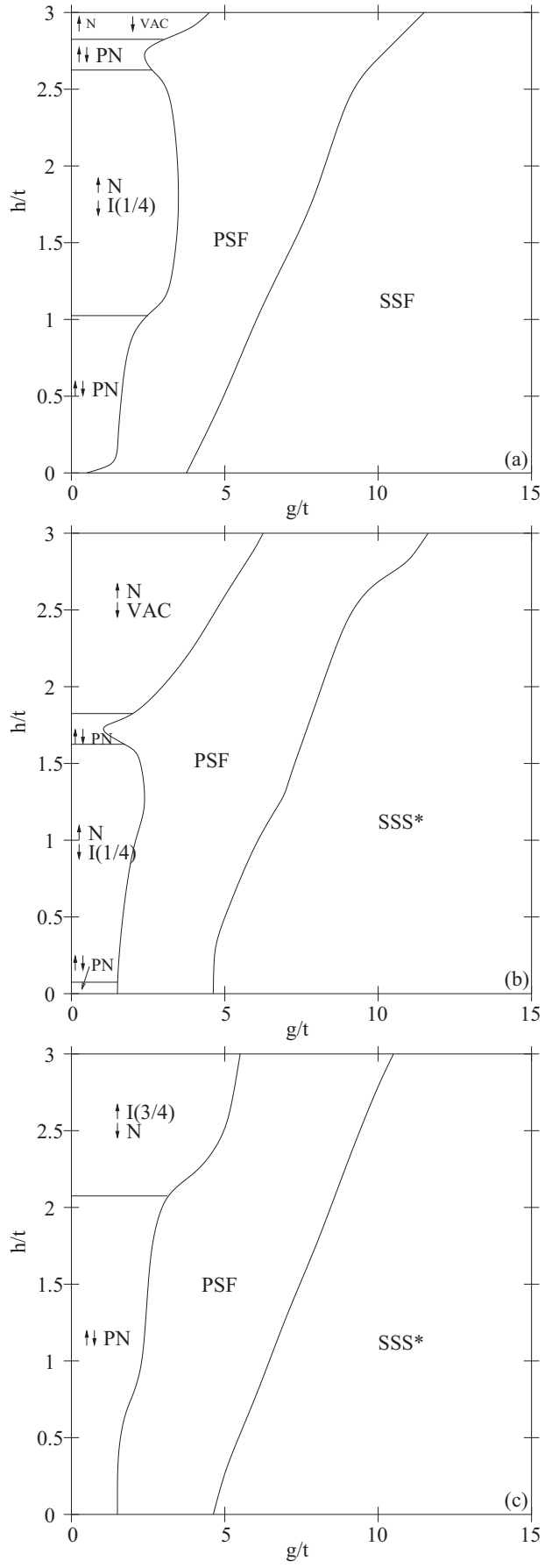

FIG. 7. Charged-uncharged mixtures of fermions: $\phi_{\uparrow}=0$ and $\phi_{\downarrow}=1 / 4$ case in (a),(b) and $\phi_{\uparrow}=1 / 4$ and $\phi_{\downarrow}=0$ case in (c). The ground-state phase diagrams are shown for $\mu=0$ in (a) and $\mu=-t$ in (b) and (c). Thanks to the particle-hole symmetry, the $\phi_{\uparrow}=1 / 4$ and $\phi_{\downarrow}=0$ phase diagram for $\mu=0$ can easily be deduced from (a) via $\uparrow \rightarrow \downarrow$ and $\downarrow \rightarrow \uparrow$.

e.g., a PDW order [27]. The directions of center-of-mass momenta determine the direction of modulations, making it gauge dependent, e.g., the $y$ direction in Fig. 2. When the striped-PDW order is sufficiently large, it drives an additional striped-CDW order in the total fermion filling, giving rise to striped-SS phases.
We emphasize that the instabilities towards stripe-ordered phases discussed in this paper are driven by the gauge fields, and they may not formally be identified with the FFLO phase which is driven by the Zeeman field and is characterized by cosinelike sign-changing $\left|\Delta_{i}\right|$ oscillations along a spontaneously chosen direction [28-30]. In addition, while the periods of our striped-PDW, -CDW, and -SDW orders are always given by $q_{d}$, the period of FFLO modulations is determined by the mismatch $h$ between $\uparrow$ and $\downarrow$ Fermi surfaces. For instance, when $\phi_{\uparrow}=\phi_{\downarrow}=p / q$, the stripes have a spatial period of $q$ or $q / 2$ lattice sites, depending on whether $q$ is odd or even. Lastly, while our striped phases survive even in the extreme dimer-BEC limit $(g / t \gg 1)$ for a large parameter space, the FFLO modulations survive not only in the BCS limit but also for a tiny parameter space near the PSF to $N$ transition boundary.

\section{CONFINED ATOMIC SYSTEMS}

Having explored the ground states and phase diagrams of thermodynamic systems, here we study confined systems and comment on the likelihood of observing stripe-ordered phases by loading neutral atomic Fermi gases on laser-induced optical lattices under laser-generated artificial gauge fields. For this purpose, we consider a harmonically confined $51 \ell \times 51 \ell$ square lattice with an isotropic trapping potential $V_{i}=\alpha\left|\mathbf{r}_{i}\right|^{2}$ centered at the origin, where $\alpha=0.01 t / \ell^{2}$ is its strength and $\mathbf{r}_{i} \equiv\left(i_{x}, i_{y}\right)$ is the position of site $i$.

\section{A. Effects of harmonic confinement}

The local ground states of trapped systems can be reliably inferred through the so-called local-density approximation, where the local density of the system is mapped to that of a thermodynamic one with the same density. This description is known to be very accurate for large systems that are trapped in slowly varying potentials. For our model Hamiltonian, due to the energy gaps of the HB and the Pauli exclusion principle, one expects so-called wedding-cake structures in the $n_{i \uparrow}$ and $n_{i \downarrow}$ profiles of noninteracting fermions at $T=0$, where the number of minigaps determines the number of spatially flat $n_{i \sigma}$ regions for a given $\phi_{\sigma}$. Thus, wedding-cake structures consist of a number of insulating regions that are sandwiched between normal regions. However, since the majority of these minigaps are very small compared to $t$, finite $g$ and/or finite $T$ quickly smear out the flat regions, making their detection nearly impossible. In sharp contrast, here we show that the broken spatial symmetry and stripe orders persist at intermediate and strong interactions, providing a viable knob for the experimental probe of the fractal structure of the HB.

In Fig. 8, we illustrate a typical self-consistent solution for a trapped system when $\phi_{\uparrow}=1 / 4, \phi_{\downarrow}=0, \mu=t, h=0$, and $g=5 t$. The total numbers of $\sigma$ fermions are approximately given by $N_{\uparrow}=N_{\downarrow} \approx 464$. While the remnants of the so-called wedding-cake structure, i.e., spatially flat $n_{i \uparrow}$ regions around integer multiples of $1 / 4$ fillings, are hardly recognizable, a large PDW order is clearly visible. Given the phase diagrams discussed in Sec. IVC, both CDW and SDW orders are expected to be weak around half filling, since $n_{i \uparrow}+n_{i \downarrow} \gtrsim 1$ near the center of the trap for this particular set of data. 

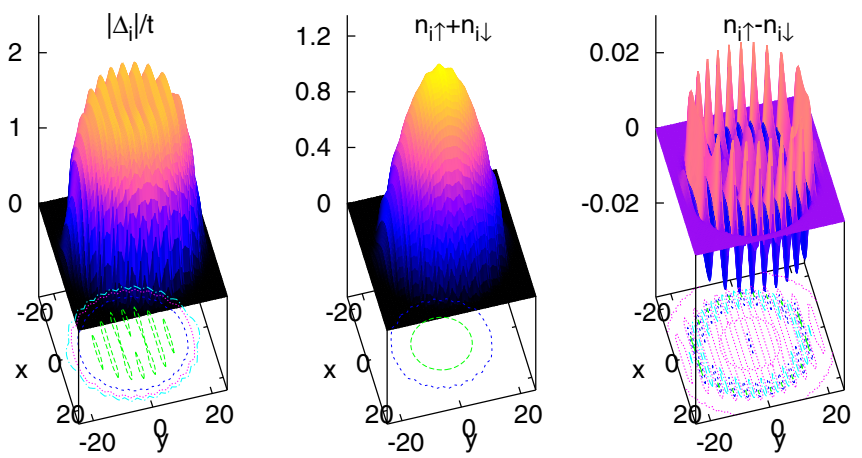

FIG. 8. (Color online) The trap profiles for $\phi_{\uparrow}=1 / 4, \phi_{\downarrow}=0$, $\mu=t, h=0$, and $g=5 t$. Here, $(x, y)$ are in units of $\ell$.

It is easier to visualize and present such trap profiles for a cut along the $y$ direction at a particular $x$ value. For instance, we show $x=0$ cuts in Figs. 9 and 10, where $\phi_{\uparrow}=1 / 4, \mu=t$, and $h=0$ in both figures, but $\phi_{\downarrow}=1 / 4$ and $\phi_{\downarrow}=0$, respectively. While the local ground states are always unpolarized in Fig. 9 where $n_{i \uparrow}=n_{i \downarrow}$ for every $i$, the imbalance between $\phi_{\uparrow}$ and $\phi_{\downarrow}$ causes small but visible SDW orders in Fig. 10. We note that $\phi_{\uparrow} \neq \phi_{\downarrow}$ may also cause a global polarization, i.e., $N_{\uparrow} \neq$ $N_{\downarrow}$, for weak $g$; however, this polarization must gradually disappear towards the dimer-BEC limit. For instance, as $g / t$ increases to $(4,5,6,7)$, while $N_{\uparrow}=N_{\downarrow}$ is approximately given by $(454,468,491,519)$ in Fig. $9, N_{\uparrow}$ and $N_{\downarrow}$ are given, respectively, by $(456,464,489,518)$ and $(448,464,489,518)$ in Fig. 10.

These figures show that the CDW and SDW orders tend to be more prominent for intermediate $g$ as long as the system is away from half filling. This is quite intuitive since the appearance of a PDW order breaks the spatial symmetry of the system in the first place. The spatial periods are, respectively, given by two and four sites in Figs. 9 and 10, and these findings are in agreement with our analysis given in Sec. III B. In addition, since the stripes eventually fade away towards the dimer-BEC limit, the trap profiles slowly recover the usual

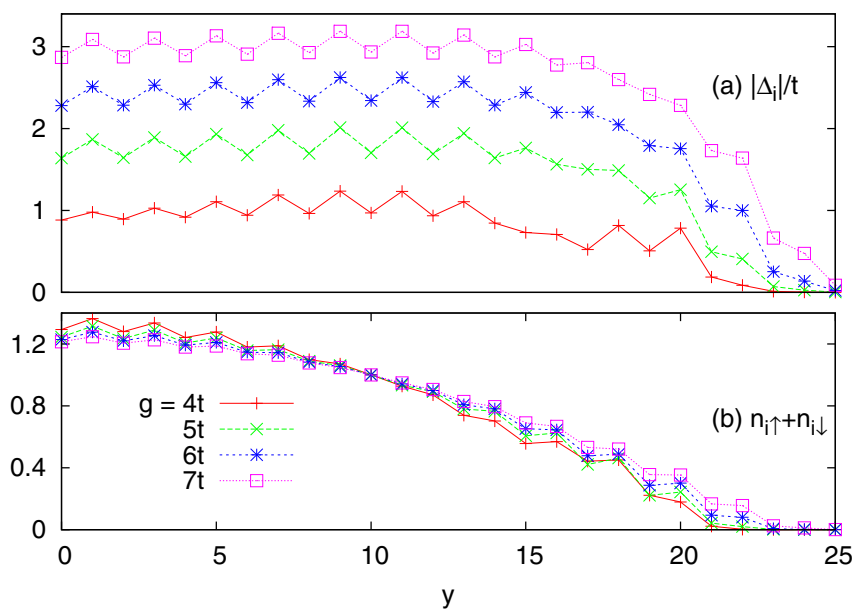

FIG. 9. (Color online) The trap profiles for $x=0$ cuts along the $y$ direction which is in units of $\ell$. Here, $\phi_{\uparrow}=\phi_{\downarrow}=1 / 4, \mu=t$, and $h=0$, and therefore, the system is locally unpolarized at every $i$.

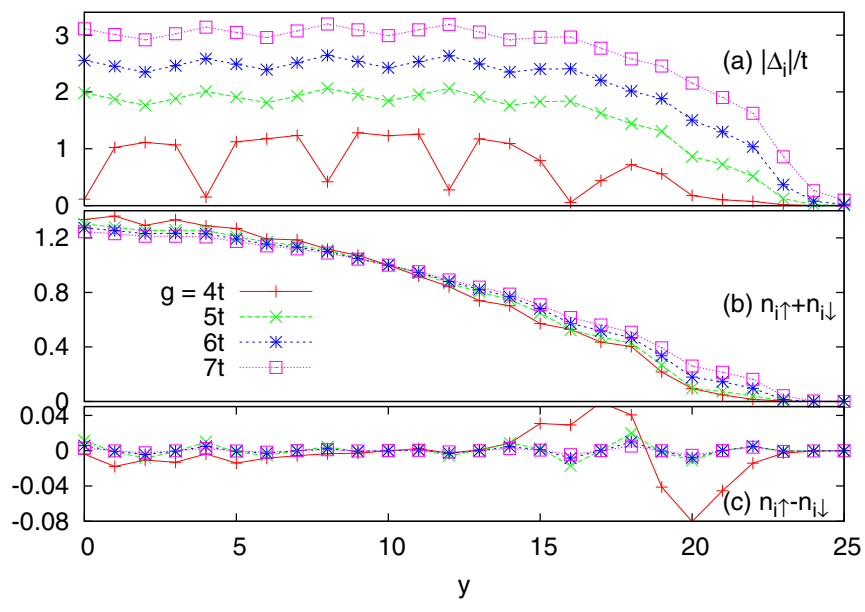

FIG. 10. (Color online) The trap profiles for $x=0$ cuts along the $y$ direction which is in units of $\ell$. Here, $\phi_{\uparrow}=1 / 4, \phi_{\downarrow}=0, \mu=t$, and $h=0$.

(no-gauge-field) results in both figures. It is also pleasing to see that the valleys of the PDW and CDW orders and peaks of the SDW order coincide when they coexist. These results suggest that observation of PDW, CDW, and SDW features as functions of magnetic flux may furnish the clearest and most direct evidence for the existence of multiple band structure, and hence indirectly for the fractal $\mathrm{HB}$, in trapped atomic systems.

\section{B. Effects of Hartree shifts}

Since most of our phases have coexisting CDW and/or SDW orders, our phase diagrams may not be convenient for generating more accurate phase diagrams by including the Hartree terms via a simple shift in $\mu_{i \sigma}$. However, we still neglected these shifts in our diagrams for their numerical as well as analytical simplicity. For instance, including these shifts in the self-consistency Eqs. (6)-(9) not only requires about an order of magnitude more iterations to converge, but also complicates our current intuition, making it more difficult to extract the relation between the HB and the nonmonotonic dependences of some of the phase boundaries. Note that, since Hartree shifts have no role in driving the stripe-ordered phases, which is particularly clear in the dimer-BEC limit where the $\mu_{i \sigma}$ do not explicitly play any role in our analysis, their inclusion is expected to change some of the transition boundaries without much effect on the stability of phases. Furthermore, since the mean-field theory provides only a qualitative description of the phase diagrams and the accuracy of our results can be somewhat improved by including these shifts, one still needs to go beyond this approximation for experimentally more relevant diagrams. Therefore, even though Hartree shifts are neglected in Sec. IV, our results may already pave the way to qualitative understanding of the exact ground states of the attractive Hofstadter-Hubbard model.

To illustrate these points, the Hartree-shifted trap profiles are shown in Figs. 11 and 12 for the parameters of Figs. 9 and 10 , respectively. Comparing these figures shows that while the inclusion of the Hartree shifts does not have much effect on 


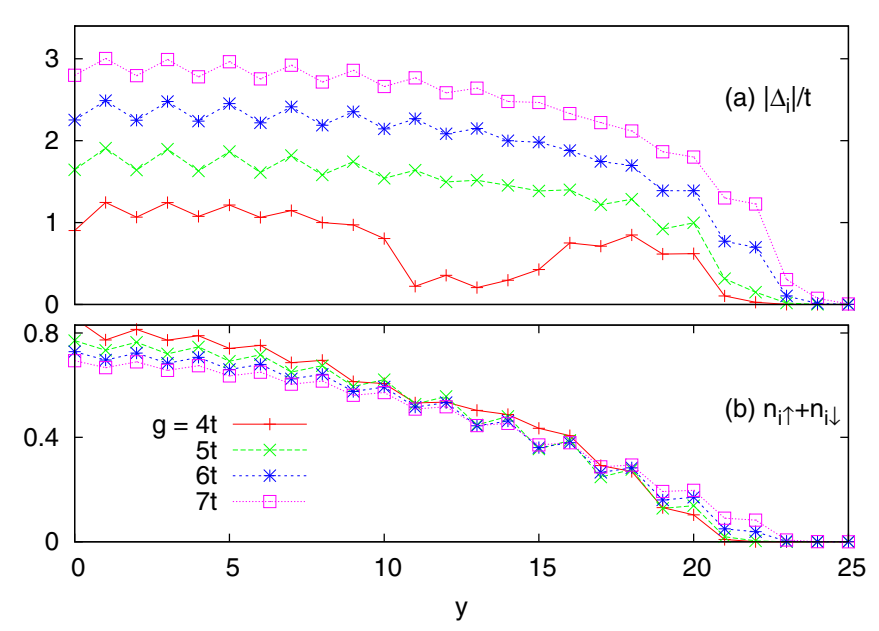

FIG. 11. (Color online) The trap profiles for $x=0$ cuts along the $y$ direction which is in units of $\ell$. Here, $\phi_{\uparrow}=\phi_{\downarrow}=1 / 4, \mu=t$, and $h=0$, i.e., the same as Fig. 9 with the Hartree shifts included.

$\left|\Delta_{i}\right|$ for these particular sets of data (thanks to the particle-hole symmetry around half filling), it affects the total filling quite a bit. For instance, as $g / t$ increases to $(4,5,6,7)$, while $N_{\uparrow}=N_{\downarrow}$ is approximately given by $(288,279,279,286)$ in Fig. $11, N_{\uparrow}$ and $N_{\downarrow}$ are given, respectively, by $(298,278,278,285)$ and $(276,282,278,285)$ in Fig. 12. However, the visibility of the striped-PDW and -CDW orders remains largely the same in both cases. In addition, we note that the remnants of the wedding-cake structures, i.e., spatially flat $n_{i \uparrow}+n_{i \downarrow}$ regions around 1/2 filling, are almost recognizable in Figs. 11(a) and 12(a) when $g=4 t$ or less (not shown). While the noninteracting $\uparrow$ fermions are insulating at $1 / 4$ filling in both figures, the noninteracting $\downarrow$ fermions are insulating (normal) in Fig. 11 (12). Thus, these insulating regions leave their traces as distinct $\left|\Delta_{i}\right|$ dips in both figures near $y=13 \ell$ when $g$ is sufficiently weak.

Given these numerical illustrations, it is clear that our phase diagrams already shed some light on an additional stripe mechanism in the dimer-BEC limit, showing that the fates of

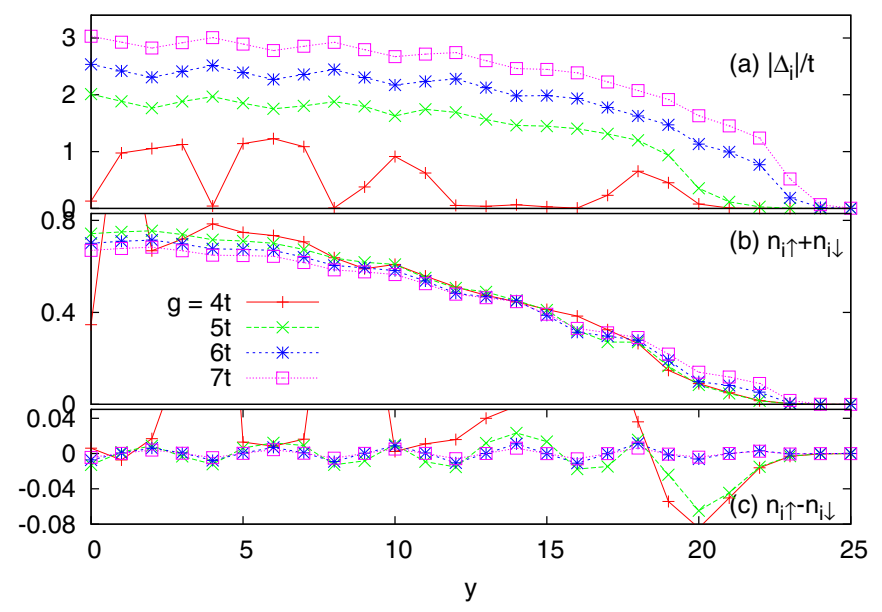

FIG. 12. (Color online) The trap profiles for $x=0$ cuts along the $y$ direction which is in units of $\ell$. Here, $\phi_{\uparrow}=1 / 4, \phi_{\downarrow}=0, \mu=t$, and $h=0$, i.e., the same as in Fig. 10 with the Hartree shifts included. stripe-ordered SF and SS phases are not affected by the Hartree terms. Having discussed the effects of confinement potentials, we are ready to end the paper with a brief summary of our conclusions and an outlook.

\section{CONCLUSIONS}

Our mean-field results for the attractive single-band Hofstadter-Hubbard model on a square lattice are as follows. In the presence of a Zeeman field $h$, in addition to the intriguing phase transition boundaries between the $N, I$, vac, and SF phases, we found a number of distinct many-body phases which can be characterized with respect to their coexisting striped-PDW, -CDW and -SDW orders. Even at $h=0$, we reached four important conclusions. First, we numerically found an unpolarized striped-superfluid phase (SSF) in a large parameter space. Unlike the conventional FFLO phase which is driven by $h$, our SSF is driven only by the gauge fields. Second, we numerically found an unpolarized striped-supersolid phase (SSS) in a large parameter space. Unlike the conventional SS phase which is yet to be observed and is driven either by longrange (e.g., nearest-neighbor) interactions or by the presence of a second species (e.g., Bose-Fermi or Bose-Bose mixtures), our SSS is again driven only by the gauge fields. Third, we also found a locally polarized but globally unpolarized striped-SS phase (SSS*) when the gauge fields are imbalanced. Lastly, we provided analytical insights into the microscopic origins of these stripe-ordered phases, suggesting a physical mechanism that gives rise to FFLO-like SF and SS phases in the dimer-BEC limit.

The importance of these results can be highlighted as follows. First, spatially modulated SF and SS phases are both of high interest not only to the atomic physics community but also to the condensed-matter, nuclear, and elementary-particle physics communities. Second, the unusual appearance of the stripe order is very exotic and fundamentally important by itself, because the connection between the striped-charge order that is observed in copper oxide materials and the formation of high- $T_{c}$ superconductivity has been the subject of a long debate in the literature. Even though our work offers no direct relation to cuprate superconductors, understanding stripe-ordered phases in the cold-atom context may still prove to be beneficial for the high- $T_{c}$ community. Third, the existence of stripe-ordered phases is not an artifact of our mean-field $\mathrm{BdG}$ description, since they are analytically motivated in the dimer-BEC limit. Therefore, we highly encourage further research in this direction with different lattice geometries, gauge fields, etc., in particular those beyond the mean field.

\section{ACKNOWLEDGMENTS}

The author thanks Dr. R. O. Umucalilar for discussions. This work is supported by TÜBITAK Grant No. 1001114F232.

\section{APPENDIX: DIMER-BEC LIMIT IN THE SYMMETRIC GAUGE}

It may be important to remark here that the analysis given in Sec. III B depends on the particular artificial gauge field 

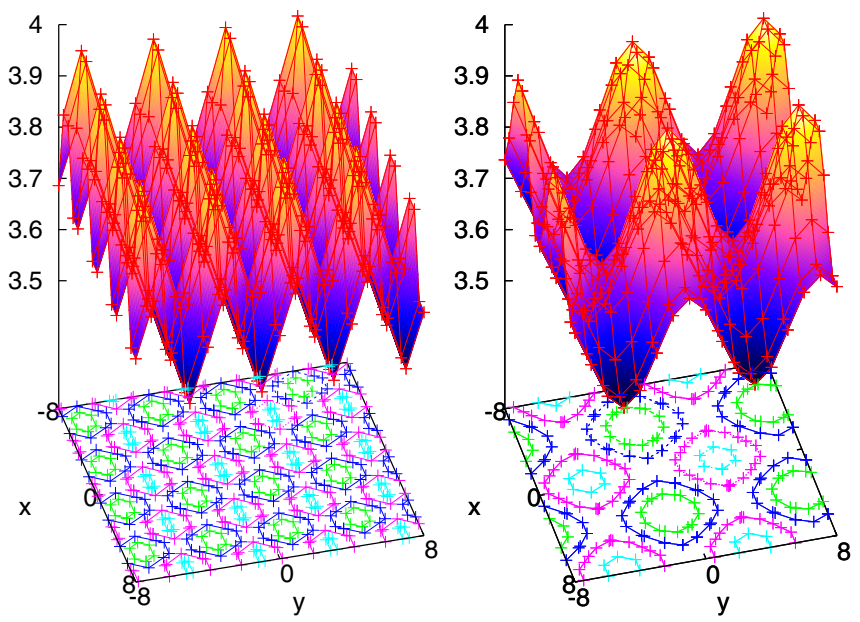

FIG. 13. (Color online) Typical $\left|\Delta_{i}\right| / t$ profiles for the checkerboardlike stripes, where $\phi_{\uparrow}=\phi_{\downarrow}=1 / 4$ in the left and $1 / 8$ in the right figure as determined by the symmetric gauge. Here, $(x, y)$ are in units of $\ell$, and we set $\mu=0, h=0$, and $g=8 t$ in both figures.

that is simulated in a cold-atom experiment. Next, we use a rotationally invariant symmetric gauge for the vector potential, i.e., $\mathbf{A}_{\sigma}(\mathbf{r}) \equiv B_{\sigma}(-y, x, 0) / 2$, and analyze the spatial structure of the order parameter $\left|\Delta_{i}\right|$ in the dimer-BEC limit, which clearly reveals this dependence.

Typical $\left|\Delta_{i}\right|$ profiles are illustrated in Fig. 13 for $\phi_{\sigma}=$ $1 / 4$ and $1 / 8$, i.e., $\phi_{d}=\phi_{\uparrow}+\phi_{\downarrow} \equiv p_{d} / q_{d}$ is $1 / 2$ and $1 / 4$, respectively. Since the $C_{4}$ symmetry of the square lattice is preserved in this gauge, the stripes are checkerboardlike in the $(x, y)$ plane, such that generalization of Eq. (10) to

$$
\left|\Delta_{i}\right|=\left|\Delta_{0}\right|+\left|\Delta_{1}\right|\left[2-\cos \left(\pi \phi_{d} i_{x} / \ell\right)-\cos \left(\pi \phi_{d} i_{y} / \ell\right)\right]
$$

fits very well with all of our numerical results in the dimer-BEC limit. Here, the uniform contribution $\left|\Delta_{0}\right|=$ $\left(g / 2-4 t^{2} / g\right) \sqrt{n(2-n)}$ is determined by the total average filling $n$ with $\mu=\left(g / 2-8 t^{2} / g\right)(n-1)$ [26], $\left|\Delta_{1}\right| \approx t^{2} / g$ for $\mu \approx 0$, and $\mathbf{r}_{i} \equiv\left(i_{x}, i_{y}\right)$ is the position of site $i$. We again note that this analytical expression is consistent with our expectation that, while the dimer-BEC order parameter in principle has contributions from all degenerate momenta $\Psi_{i d}=$ $\sum_{\mathbf{k}_{d}} c_{\mathbf{k}_{d}} e^{i \mathbf{k}_{d} \cdot \mathbf{r}_{i}}$, where $c_{\mathbf{k}_{d}}=\left|c_{\mathbf{k}_{d}}\right| e^{i \vartheta_{\mathbf{k}_{d}}}$ are complex variational parameters, only the lowest-order $\mathbf{k}_{d}=\left\{(0,0) ; \pi \phi_{d}\left(f_{x}, f_{y}\right) / \ell\right\}$ terms contribute when $g / t$ is sufficiently large. This is because dimer bosons are fermion pairs and the number of ways of creating them with $\mathbf{k}_{d}=\mathbf{k}_{\uparrow}+\mathbf{k}_{\downarrow}$ momentum depends on $f_{x}$, $f_{y}, \phi_{\uparrow}$, and $\phi_{\downarrow}$, and higher-k $\mathbf{k}_{d}$ states contribute less and less, forming again a perturbative series.

In addition, moving towards the BCS side, the second-order corrections to Eq. (A1) can be shown to be $+\left|\Delta_{2}\right|\left[\cos \left(2 \pi \phi_{d} i_{x} / \ell\right)+\cos \left(2 \pi \phi_{d} i_{y} / \ell\right)\right]$ and $+\left|\Delta_{2}^{\prime}\right|$ $\cos \left(\pi \phi_{d} i_{x} / \ell\right) \cos \left(\pi \phi_{d} i_{y} / \ell\right)$ for any $q_{d}$. Since these terms are in ( $\pi$ out of ) phase with the zeroth- (first-) order term, they tend to create dimples at the peaks arising from the first-order one, suggesting that $\vartheta_{\mathbf{k}_{d}}-\vartheta_{\mathbf{0}}=\pi\left(f_{x}+f_{y}\right)$, i.e., the form of $\left|\Psi_{i d}\right|$ again coincides with $\left|\Delta_{i}\right|$ under these conditions. Finally, we note that both our numerical results and the analytical fit Eq. (A1) clearly show that modulations of $\left|\Delta_{i}\right|$ have a spatial period of $2 q_{d}$ lattice sites along both $x$ and $y$ directions, as expected for the resultant dimers in the symmetric gauge.
[1] D. R. Hofstadter, Phys. Rev. B 14, 2239 (1976).

[2] M. Kohmoto, Phys. Rev. B 39, 11943 (1989).

[3] C. R. Dean et al., Nature (London) 497, 598 (2013).

[4] L. A. Ponomarenko et al., Nature (London) 497, 594 (2013).

[5] V. Galitski and I. B. Spielman, Nature (London) 494, 49 (2013).

[6] J. Dalibard, F. Gerbier, and G. Juzeliŭnas, and P. Öhberg, Rev. Mod. Phys. 83, 1523 (2011).

[7] J.-Y. Zhang, S.-C. Ji, Z. Chen, L. Zhang, Z.-D. Du, B. Yan, G.-S. Pan, B. Zhao, Y.-J. Deng, H. Zhai, S. Chen, and J.-W. Pan, Phys. Rev. Lett. 109, 115301 (2012).

[8] P. Wang, Z.-Q. Yu, Z. Fu, J. Miao, L. Huang, S. Chai, H. Zhai, and J. Zhang, Phys. Rev. Lett. 109, 095301 (2012).

[9] L. W. Cheuk, A. T. Sommer, Z. Hadzibabic, T. Yefsah, W. S. Bakr, and M. W. Zwierlein, Phys. Rev. Lett. 109, 095302 (2012).

[10] C. Qu, C. Hamner, M. Gong, C. Zhang, and P. Engels, Phys. Rev. A 88, 021604(R) (2013).

[11] Z. Fu, L. Huang, Z. Meng, P. Wang, X.-J. Liu, H. Pu, H. Hu, and J. Zhang, Phys. Rev. A 87, 053619 (2013).

[12] R. A. Williams, M. C. Beeler, L. J. LeBlanc, K. Jiménez-García, and I. B. Spielman, Phys. Rev. Lett. 111, 095301 (2013).

[13] K. Jiménez-García, L. J. LeBlanc, R. A. Williams, M. C. Beeler, A. R. Perry, and I. B. Spielman, Phys. Rev. Lett. 108, 225303 (2012).

[14] J. Struck, C. Ölschläger, M. Weinberg, P. Hauke, J. Simonet, A. Eckardt, M. Lewenstein, K. Sengstock, and P. Windpassinger, Phys. Rev. Lett. 108, 225304 (2012).
[15] C. J. Kennedy, G. A. Siviloglou, H. Miyake, W. C. Burton, and W. Ketterle, Phys. Rev. Lett. 111, 225301 (2013).

[16] M. Aidelsburger, M. Atala, M. Lohse, J. T. Barreiro, B. Paredes, and I. Bloch, Phys. Rev. Lett. 111, 185301 (2013).

[17] H. Miyake, G. A. Siviloglou, C. J. Kennedy, W. C. Burton, and W. Ketterle, Phys. Rev. Lett. 111, 185302 (2013).

[18] C. Chin and E. J. Mueller, Physics 6, 118 (2013).

[19] D. Cocks, P. P. Orth, S. Rachel, M. Buchhold, K. Le Hur, and W. Hofstetter, Phys. Rev. Lett. 109, 205303 (2012).

[20] L. Wang, H.-H. Hung, and M. Troyer, Phys. Rev. B 90, 205111 (2014).

[21] Hui Zhai, R. O. Umucalılar, and M. O. Oktel, Phys. Rev. Lett. 104, 145301 (2010).

[22] R. Wei and E. J. Mueller, Phys. Rev. Lett. 108, 245301 (2012).

[23] M. Iskin, Phys. Rev. A 91, 011601(R) (2015).

[24] T. Esslinger, Annu. Rev. Condens. Matter Phys. 1, 129 (2010).

[25] S. Powell, R. Barnett, R. Sensarma, and S. Das Sarma, Phys. Rev. Lett. 104, 255303 (2010).

[26] M. Iskin and C. A. R. Sá de Melo, Phys. Rev. A 78, 013607 (2008)

[27] D. F. Agterberg and H. Tsunetsugu, Nat. Phys. 4, 639 (2008).

[28] P. Fulde and R. Ferrell, Phys. Rev. 135, A550 (1964).

[29] A. I. Larkin and Y. N. Ovchinnikov, Zh. Eksp. Teor. Fiz. 47, 1136 (1964) [Sov. Phys. JETP 20, 762 (1965)].

[30] R. Casalbuoni and G. Nardulli, Rev. Mod. Phys. 76, 263 (2004) 